\title{
THE
}

\section{Integrable motion of a vortex dipole in an axisymmetric flow}

\author{
G. G. Suyrin \\ University of Rhode Island \\ X. Perrot \\ X. Carton
}

Follow this and additional works at: https://digitalcommons.uri.edu/gsofacpubs

This is a pre-publication author manuscript of the final, published article.

Creative Commons License

(c) $($ i) $\Theta$

This work is licensed under a Creative Commons Attribution-Noncommercial-No Derivative Works 4.0 License.

\section{Citation/Publisher Attribution}

Sutyrin, G. G., Perrot, X., \& Carton, X. (2008). Integrable motion of a vortex dipole in an axisymmetric flow. Physics Letters A, 372(33), 5452-5457. doi: 10.1016/j.physleta.2008.06.038

Available at: https://doi.org/10.1016/j.physleta.2008.06.038

This Article is brought to you for free and open access by the Graduate School of Oceanography at DigitalCommons@URI. It has been accepted for inclusion in Graduate School of Oceanography Faculty Publications by an authorized administrator of DigitalCommons@URI. For more information, please contact digitalcommons-group@uri.edu. 


\title{
Integrable motion of a vortex dipole in an axisymmetric flow
}

\author{
G.G. Sutyrin ${ }^{a,{ }^{*}}$, X. Perrot ${ }^{b}$ and X. Carton ${ }^{b}$ \\ ${ }^{a}$ Graduate School od Oceanography, University of Rhode Island, Narragansett, RI 02882, USA \\ ${ }^{\mathrm{b}}$ Laboratoire de Physique des Oceans, UBO-CNRS-IFREMER, 6, avenue Le Gorgeu, 29200 Brest, France \\ *: Corresponding author : G.G. Sutyrin, Tel.: +1 401874 6213; fax: +1 401874 6728, email address : \\ gsutyrin@gso.uri.edu
}

\begin{abstract}
:
The evolution of a self-propelling vortex dipole, embedded in an external nondivergent flow with constant potential vorticity, is studied in an equivalent-barotropic model commonly used in geophysical, astrophysical and plasma studies. In addition to the conservation of the Hamiltonian for an arbitrary point vortex dipole, it is found that the angular momentum is also conserved when the external flow is axisymmetric. This reduces the original four degrees of freedom to only two, so that the solution is expressed in quadratures. In particular, the scattering of antisymmetric dipoles approaching from the infinity is analyzed in the presence of an axisymmetric oceanic flow typical for the vicinity of isolated seamounts.
\end{abstract}

Keywords: Vortex dynamics; Rotating fluids; Topography interaction; Quasi-geostrophic

PACS classification codes: 47.10.Df; 47.15.ki; 47.32.-y; 47.32.cb 


\section{Introduction}

Vortices have been recognized to be key elements in turbulent fluid motion at a wide range of scales. The Coriolis force on rotating planets or the Lorentz force due to magnetic field in plasmas makes large-scale flows anisotropic, quasi-twodimensional (e.g., the horizontal velocity of oceanic currents is much larger than vertical velocity). Coherent, long-lived vortices and jets emerge naturally as a result of self-organization of turbulent motion observed in such anisotropic media; they are well identified by the wavelet transform and Okubo-Weiss criterion, e.g. [1]. Coherent vortices are very efficient in trapping passive tracers for long times and transporting them over anomalously large distances. For this reason, mutual interaction of isolated vortices, their stability and effects of background currents have been intensively studied in the last decades as summarized in a number of reviews $[2-6]$.

Signatures of well-separated, nearly circular or elliptical, monopolar vortices of both signs are common in geophysical flows. Two vortices of opposite sign often form a self-propelling dipolar couple which provides anomalous transport of scalar properties for especially long distances. They are easily excited in twodimensional flows and they appear to be the universal outcome of an external forcing posessing a nonzero linear momentum [7-9].

Many theoretical studies of vortex couples in geophysical flows and plasmas have been essentially constrained by the $\beta$-effect which allows the permanent form solutions only with zonal direction of propagation. In the traditional quasigeostrophic approximation such steadily propagating solutions (modons as labelled by Stern) have a dipolar structure with zero net angular momentum; general solutions, including the axisymmetric rider, must also have a vanishing angular momentum in order to be stationary, i.e. the azimuthal velocity of the rider must change sign so that it is not a monopole [3]. However, zonally propagating structures on the $\beta$-plane will not lead to any meridional transport.

The variation of the intensity ratio of partners and corresponding change 
in the path curvature of non-zonally propagating dipoles have been described approximately by the well-known equation of the physical pendulum [10]. The numerical investigation of the dynamics of equivalent-barotropic $f$-plane dipoles which are steady solutions in the absence of the $\beta$-effect, demonstrated that they remain coherent on a $\beta$-plane if dipoles are strong enough [11]. Thus, the $\beta$-effect is not crucial for an intense dipole when the swirling velocity in the partners are much higher than the Rossby wave speed as observed in the upper ocean. The curved path of the vortex couple resulting from different strenths of companions may originate from the formation process and be affected only slightly by the $\beta$-effect as demonstrated in laboratory experiments [7].

The vortex evolution can be also strongly influenced by nonuniform background currents. However, the evolution of vortex dipoles in external flows have received only little attention in literature. In particular, a horizontal strain would either accelerate the dipole and form a head-tail structure, or separate the partners, depending on the strain orientation [12]. Under the influence of a radial flow from a point source (or sink), dipoles can separate, converge or follow spiraling trajectories [13].

In the present study, we consider evolution of vortex couples in a horizontally sheared nondivergent flow with constant potential vorticity. Vortex couples are considered in the point vortex approximation that allows to express the solution in quadratures for the case of axisymmetric external flow and analyze different dynamical regimes explicitly for an external flow typical for a topographic circulation around an isolated seamount [14]. The mathematical formulation is presented in Section 2. The solution in the general form for arbitrary ratio of point vortex intensities is described in Section 3. The behavior of a dipolar couple approaching from the infinity is analyzed in Section 4. Discussion and conclusions are in Section 5. 


\section{Mathematical formulation}

\subsection{Potential vorticity equation}

At the lowest order of approximation, quasi-two-dimensional dynamics of oceans, planetary atmospheres and of plasmas in a magnetic field are governed by the conservation of a material invariant $Q$ in an equivalent-barotropic model [5]

$$
\partial_{t} Q+\mathbf{u} \nabla Q=0
$$

Here $t$ is time normalized by a time scale $T,(x, y)$ are horizontal coordinates normalized by a horizontal scale $L, \mathbf{u}$ is the flow velocity normalized by $L / T$. In geophysical fluid applications such model describes a thin layer of homogeneous, incompressible fluid, strongly constrained by ambient rotation and by stratification, overlying an infinitely deep layer of fluid at rest [6]. Then potential vorticity $Q$ represents the ratio of absolute vorticity to the layer thickness and it is conserved in each fluid parcel.

For small Rossby number and order unity Burger number, the velocity is nondivergent in the leading order, so that the velocity and potential vorticity are expressed by the streamfunction, $\psi(x, y, t)$ normalized by $L^{2} / T$

$$
\mathbf{u}(x, y, t)=\mathbf{k} \times \nabla \psi, \quad Q=\nabla^{2} \psi-S,
$$

where $\mathbf{k}$ is the vertical unit vector, and the term $S$ is the vortex stretching related to changes of the layer thickness either due to localized topography $S(x, y)$, or due to geostrophic adjustment outside topography, where $S=\gamma^{2} \Psi$. Here $\gamma=f L / C_{g}$, the gravity wave speed, $C_{g}$, and the Coriolis parameter, $f$, are assumed to be constants. For the plasma case, $\psi$ is proportional to the electrostatic potential normalized by $T_{e} / e\left(T_{e}\right.$ is the electron temperature, $e$ is the electric charge of the ion), $C_{g}=\sqrt{T_{e} / m_{i}}$ is the ion sound speed $\left(m_{i}\right.$ is the ion mass), and $f=\omega_{i c}$ is the ion cyclotron frequency [15].

In the limiting case of $\gamma \rightarrow 0,(1)-(2)$ describe pure two-dimensional in- 
compressible flows and they are valid for an arbitrary Rossby number. In both models, the domain is infinite in horizontal directions.

\subsection{Point vortex model}

Following [16], we decompose the flow into a steady external part, $\mathbf{U}=\mathbf{k} \times \nabla \Psi$, and a vortical part $\mathbf{u}_{V}=\mathbf{u}-\mathbf{U}$ which corresponds to localized vortices.

Assuming that the vortex dipole is represented by localized potential vorticity anomalies with amplitudes $\kappa_{1}$ and $\kappa_{2}$, their self-induced drift affected by the external flow is governed by the following equations

$\frac{d x_{1}}{d t}=-\partial_{y} \Psi\left(x_{1}, y_{1}\right)+\kappa_{2}\left(y_{2}-y_{1}\right) \omega\left(r_{12}\right), \quad \frac{d y_{1}}{d t}=\partial_{x} \Psi\left(x_{1}, y_{1}\right)-\kappa_{2}\left(x_{2}-x_{1}\right) \omega\left(r_{12}\right)$,

$\frac{d x_{2}}{d t}=-\partial_{y} \Psi\left(x_{2}, y_{2}\right)-\kappa_{1}\left(y_{2}-y_{1}\right) \omega\left(r_{12}\right), \quad \frac{d y_{2}}{d t}=\partial_{x} \Psi\left(x_{2}, y_{2}\right)+\kappa_{1}\left(x_{2}-x_{1}\right) \omega\left(r_{12}\right)$,

where $r_{i}^{2}=x_{i}^{2}+y_{i}^{2}, r_{12}^{2}=\left(x_{1}-x_{2}\right)^{2}+\left(y_{1}-y_{2}\right)^{2}$, and $\omega(r)$ is the rotation rate of a point vortex, either in the equivalent-barotropic model, or in the 2D model:

$$
\omega(r)=\frac{d \psi}{r d r}, \quad \psi=-\frac{1}{2 \pi} K_{0}(\gamma r), \quad \text { or } \quad \psi=\frac{1}{2 \pi} \ln r
$$

The system of ODE (3)-(4) conserves the Hamiltonian $\mathcal{H}[2]$ :

$$
\mathcal{H}=\Psi\left(r_{1}\right)+q \Psi\left(r_{2}\right)+q \kappa_{1} \psi\left(r_{12}\right)=\text { const }
$$

where $q=\kappa_{2} / \kappa_{1}$ while the components of linear momentum and the total angular momentum, $M$, evolve due to the external flow:

$$
\begin{gathered}
\dot{x}_{1}+q \dot{x}_{2}=-\partial_{y} \Psi\left(x_{1}, y_{1}\right)-q \partial_{y} \Psi\left(x_{2}, y_{2}\right), \quad \dot{y}_{1}+q \dot{y}_{2}=\partial_{x} \Psi\left(x_{1}, y_{1}\right)+q \partial_{x} \Psi\left(x_{2}, y_{2}\right), \\
\dot{M}=\left(y_{1} \partial_{x}-x_{1} \partial_{y}\right) \Psi\left(x_{1}, y_{1}\right)+q\left(y_{2} \partial_{x}-x_{2} \partial_{y}\right) \Psi\left(x_{2}, y_{2}\right), \quad 2 M=r_{1}^{2}+q r_{2}^{2},
\end{gathered}
$$

Therefore, analytical solutions for point vortex evolution in an external flow 
were found only in cases with additional symmetry considered in [13].

As one can see from (8), the total angular momentum is conserved $M=$ const when the external flow is axisymmetric and stationary on the $f$-plane,

$$
U=-y \Omega(r), \quad V=x \Omega(r), \quad \Omega=\frac{d \Psi}{r d r},
$$

where $\Omega$ is the external rotation rate and $r^{2}=x^{2}+y^{2}$.

Conservation of total angular momentum in this case, added to the Hamiltonian conservation, allows a reduction from the original four degrees of freedoms to only two and a general solution in quadratures.

\section{Motion of a vortex dipole in the general case}

\subsection{Simple case of constant external rotation rate}

When $\Omega=$ const, the solution is obvious in coordinates rotating with the angular rate $\Omega$ : the distance between partners is known to remain constant $r_{12}=$ const, while the center of the dipole defined as $x_{c}=\left(x_{1}+x_{2}\right) / 2, y_{c}=\left(y_{1}+y_{2}\right) / 2$ moves along circular trajectories with radius $r_{q}=r_{12}(1-q) /[2(1+q)]$. In particular, equal vortices of the same sign $(q=1)$ rotate around each other $\left(r_{q}=0\right)$, while a dipole with opposite sign vortices $(q=-1)$ propagates uniformly $\left(r_{q}=\infty\right)$ with the speed $U_{\infty}=r_{12} \omega\left(r_{12}\right)$.

\subsection{Integrability of vortex motion in the general case}

In the general case of an external flow with differential rotation, we can choose a reference frame such that $\Omega \rightarrow 0$ and $\Psi \rightarrow 0$ at infinity $(r \rightarrow \infty)$. The trajectory of the dipole center is described by

$$
\dot{x}_{c}=-\frac{y_{1} \Omega\left(r_{1}\right)+y_{2} \Omega\left(r_{2}\right)}{2}-\frac{1-q}{2}\left(y_{2}-y_{1}\right) \omega\left(r_{12}\right),
$$




$$
\dot{y}_{c}=\frac{x_{1} \Omega\left(r_{1}\right)+x_{2} \Omega\left(r_{2}\right)}{2}+\frac{1-q}{2}\left(x_{2}-x_{1}\right) \omega\left(r_{12}\right)
$$

Here the time scale $T$ is chosen such that $\kappa_{1}=1$.

Using the variables $r_{c}=\left(x_{c}^{2}+y_{c}^{2}\right)^{1 / 2}$ and $\theta_{c}=\operatorname{atan}\left(y_{c} / x_{c}\right)$, the system (10)-(11) can be rewritten as

$$
\begin{gathered}
\dot{r}_{c}=-\frac{y_{1} \Omega\left(r_{1}\right)+y_{2} \Omega\left(r_{2}\right)}{2} \cos \theta_{c}+\frac{x_{1} \Omega\left(r_{1}\right)+x_{2} \Omega\left(r_{2}\right)}{2} \sin \theta_{c}+U \sin \left(\theta_{c}-\theta_{12}\right) \\
r_{c} \dot{\theta}_{c}=\frac{x_{1} \Omega\left(r_{1}\right)+x_{2} \Omega\left(r_{2}\right)}{2} \cos \theta_{c}+\frac{y_{1} \Omega\left(r_{1}\right)+y_{2} \Omega\left(r_{2}\right)}{2} \sin \theta_{c}+U \cos \left(\theta_{c}-\theta_{12}\right) .
\end{gathered}
$$

where $U\left(r_{12}\right)=(1-q) r_{12} \omega\left(r_{12}\right) / 2$ is the drift velocity due to vortex interaction inside the dipole, and the angle $\theta_{12}$ characterizes its orientation $\theta_{12}=\operatorname{atan}\left(\left(y_{2}-\right.\right.$ $\left.\left.y_{1}\right) /\left(x_{2}-x_{1}\right)\right)$ : when $\theta_{12}=\theta_{c}$ the self-induced drift velocity is in the azimuthal direction, when $\theta_{12}=\theta_{c} \pm \pi / 2$ the self-induced drift is in the radial direction (see Fig.1).

Using the expressions $x_{1}=x_{c}-\frac{r_{12}}{2} \cos \theta_{12}, x_{2}=x_{c}+\frac{r_{12}}{2} \cos \theta_{12}, y_{1}=$ $y_{c}-\frac{r_{12}}{2} \sin \theta_{12}, y_{2}=y_{c}+\frac{r_{12}}{2} \sin \theta_{12}$, we obtain

$$
\begin{gathered}
\dot{r}_{c}=U_{\text {eff }} \sin \left(\theta_{c}-\theta_{12}\right), \quad \dot{\theta}_{c}=\frac{\Omega\left(r_{1}\right)+\Omega\left(r_{2}\right)}{2}+\frac{U_{e f f}}{r_{c}} \cos \left(\theta_{c}-\theta_{12}\right), \\
r_{1}^{2}=r_{c}^{2}+\frac{r_{12}^{2}}{4}-r_{c} r_{12} \cos \left(\theta_{c}-\theta_{12}\right), \quad r_{2}^{2}=r_{c}^{2}+\frac{r_{12}^{2}}{4}+r_{c} r_{12} \cos \left(\theta_{c}-\theta_{12}\right),
\end{gathered}
$$

where $U_{\text {eff }}=U+r_{12}\left(\Omega\left(r_{2}\right)-\Omega\left(r_{1}\right)\right) / 4$ includes the additional effect of differential rotation in the external flow. For vortices of unequal strength $(q \neq 1)$, the conservation of the angular momentum allows one to express the relative angle $\theta_{c}-\theta_{12}, r_{1}$ and $r_{2}$ in terms of $r_{c}$ and $r_{12}$, via

$$
\begin{gathered}
-(1-q) \cos \left(\theta_{c}-\theta_{12}\right)=\frac{2 M}{r_{c} r_{12}}-\frac{1+q}{r_{c} r_{12}}\left(r_{c}^{2}+\frac{r_{12}^{2}}{4}\right), \\
\frac{1-q}{2} r_{1}^{2}=-q\left(r_{c}^{2}+\frac{r_{12}^{2}}{4}\right)+M, \quad \frac{1-q}{2} r_{2}^{2}=r_{c}^{2}+\frac{r_{12}^{2}}{4}-M,
\end{gathered}
$$


while the Hamiltonian provides a supplementary relation to simplify $r_{12}$ via

$$
q \psi\left(r_{12}\right)=\mathcal{H}-\Psi\left(r_{1}\right)-q \Psi\left(r_{2}\right)
$$

Finally, the system (14) can be integrated in the form

$$
\theta_{c}=-\int_{r_{c}}^{r_{0}}\left[\frac{\Omega\left(r_{1}\right)+\Omega\left(r_{2}\right)}{2 U_{e f f} \sin \left(\theta_{c}-\theta_{12}\right)}+\frac{\cot \left(\theta_{c}-\theta_{12}\right)}{r_{c}}\right] d r_{c} .
$$

setting as initial values, $\theta_{c}=0$ at $r_{c}=r_{0}$.

\subsection{Special case of co-rotating vortices}

In the case of co-rotating vortices, $q=1$, and their center rotates in the external flow with only slight variations of $r_{c}$ which can be estimated from (17)-( 18)

$r_{c}^{2}+\frac{r_{12}^{2}}{4}=r_{0}^{2}+\frac{1}{4}, \quad \psi\left(r_{12}\right)=\psi(1)+2 \Psi\left(\sqrt{r_{0}^{2}+1 / 4}\right)-\Psi\left(\left|r_{c}-r_{12}\right|\right)-\Psi\left(r_{c}+r_{12}\right)$,

The horizontal scale is chosen equal to the initial distance between vortices when $r_{1}=r_{2}=\sqrt{r_{0}^{2}+1 / 4}$.

\section{Evolution of an antisymmetric vortex dipole}

In the case of antisymmetric dipole $(q=-1)$, the integrals (16)-(18) are simplified into

$$
\begin{gathered}
-\cos \left(\theta_{c}-\theta_{12}\right)=\frac{M}{r_{c} r_{12}}, \quad r_{1}^{2}=r_{c}^{2}+\frac{r_{12}^{2}}{4}+M, \quad r_{2}^{2}=r_{c}^{2}+\frac{r_{12}^{2}}{4}-M, \\
\psi\left(r_{12}\right)=\psi(1)+\Psi\left(r_{1}\right)-\Psi\left(r_{2}\right) .
\end{gathered}
$$

where the horizontal scale is chosen equal to the distance between vortices at infinity $r_{0} \rightarrow \infty$ where $\theta_{12}=\pi / 2$ and $\mathcal{H}=q \psi(1) . M$ represents the opposite horizontal offset of the dipole center at infinity. 


\subsection{Topographically induced external axisymmetric flow}

Further we consider an example of external flow around an axisymmetric topographic feature with $S=D(r)$, localized inside the radius $r_{D}$. This external flow has uniformly null potential vorticity $(\Gamma=0)$, and, for $r \leq r_{D}$, its streamfunction $\Psi$ is obtained from

$$
\nabla^{2} \Psi=D(r), \quad \Omega(r)=\frac{1}{r^{2}} \int_{0}^{r} D\left(r^{\prime}\right) r^{\prime} d r^{\prime}
$$

and for $r>r_{D}$ by the inversion of the Helmholtz operator in the equivalentbarotropic model (with continuity of streamfunction at $r=r_{D}$ ) :

$$
\left(\nabla^{2}-\gamma^{2}\right) \Psi=0, \quad \Psi=-\Omega_{D} K_{0}(\gamma r), \Omega=\frac{\gamma \Omega_{D}}{r} K_{1}(\gamma r), \quad \Omega_{D}=\frac{r_{D} \Omega\left(r_{D}\right)}{\gamma K_{1}\left(\gamma r_{D}\right)}
$$

where $K_{j}$ is the modified Bessel function of the $j$-th order, and where $\Omega\left(r_{D}\right)$ is given by (23). For 2D incompressible flows, the stramfunction for $r>r_{D}$ is obtained by the inversion of the Poisson operator

$$
\nabla^{2} \Psi=0, \quad \Psi=\Omega_{D} \ln (r), \quad \Omega=\frac{\Omega_{D} r_{D}^{2}}{r^{2}}, \quad \Omega_{D}=\Omega\left(r_{D}\right) r_{D}^{2}
$$

\subsection{Zero angular momentum}

In the particular case where $M=0$, considerable simplification occurs: $\cos \left(\theta_{c}-\theta_{12}\right)=0$, so that $\theta_{12}=\theta_{c}+\pi / 2$ when the dipole propagates towards the plane center or $\theta_{12}=\theta_{c}-\pi / 2$ when the dipole propagates away from the center. In this case $r_{12}=1$ does not change as seen from (22) since $r_{2}=r_{1}=$

$\sqrt{r_{c}^{2}+1 / 4}$. Then, the effective drift velocity is $U_{\text {eff }}=U=\gamma K_{1}(\gamma) / 2 \pi$ and the integral (19) simplifies into

$$
\theta_{c}\left(r_{c}\right)=\frac{1}{U} \int_{r_{c}}^{\infty} \Omega\left(\sqrt{r^{2}+1 / 4}\right) d r
$$


Then, the minimum value of $r_{c}$ is zero and if $r_{D}<1 / 2$, we obtain from (24) that the dipole center passes the plane center at the angle

$$
\theta_{0}=\frac{2 \pi \Omega_{D}}{K_{1}(\gamma)} \int_{0}^{\infty} K_{1}\left(\gamma \sqrt{r^{2}+1 / 4}\right) \frac{d r}{\sqrt{r^{2}+1 / 4}}=2 \pi^{2} \Omega_{D} F(\gamma)
$$

where the coefficient $F$ only slightly depends on $\gamma: \quad F \rightarrow 1$ when $\gamma \rightarrow 0$ and $F \approx 1.1$ when $\gamma=1$ because both $U$ and $\Omega$ in (26) decrease when $\gamma$ increases.

After the dipole passes the plane center, the angle changes by $-\pi \theta_{0} /\left|\theta_{0}\right|$ (to remain in $[-\pi, \pi])$, and its subsequent values are, as the dipole moves away from the center from the plane center (see Fig.2).

$$
\theta_{c}\left(r_{c}\right)=\theta_{0}\left(1-\frac{\pi}{\left|\theta_{0}\right|}\right)+\frac{1}{U} \int_{0}^{r_{c}} \Omega\left(\sqrt{r^{2}+1 / 4}\right) d r .
$$

Thus, at infinity, the dipole propagates at the angle $\theta_{0}\left(2-\pi /\left|\theta_{0}\right|\right)$, which can take any value in $[-\pi, \pi]$, depending on the flow parameters. In particular, the dipole returns back in the original direction if $\left|\theta_{0}\right|=\pi / 2+k \pi, \quad k=0,1,2 \ldots$

\subsection{Non-zero angular momentum}

When $M<0$, and considering that the dipole drifts towards the plane center from $x \rightarrow+\infty$ (that is $\theta_{c} \sim 0$ and $\theta_{12} \sim \pi / 2$ at least initially), equation (21) and the initial conditions indicate that $\cot \left(\theta_{c}-\theta_{12}\right)<0$. Therefore, the selfinteraction of the vortices described by the second term in (19), contributes to an increase in angle $\theta_{c}$ as $r_{c}$ decreases. Conversely, for $M>0$, this selfinteraction induces an increase in angle $\theta_{c}$ decreases as $r_{c}$ decreases. In both cases, the external rotation, described by the first term in (19), results either in an additional increase of the angle $\theta_{c}$ for cyclonic rotation $\Omega>0$ or in an additional decrease of the angle $\theta_{c}$ for anticyclonic rotation $\Omega<0$, as long as $\sin \left(\theta_{c}-\theta_{12}\right)$ remains negative and $U_{\text {eff }}>0$ (assuming that $r_{1} \sim r_{2}$ ).

The radial distance $r_{c}$ reaches a minimum value $r_{m}$ when $\theta_{c}$ approaches the value $\theta_{m}=\theta_{c}\left(r_{m}\right)$ where $\sin \left(\theta_{c}-\theta_{12}\right)=0$ and $\cos \left(\theta_{c}-\theta_{12}\right)=M /|M|$. Note that 
$\left|\theta_{c}-\theta_{m}\right|$ is proportional to $\left|r_{c}-r_{m}\right|^{1 / 2}$ in the vicinity of $r_{m}$, so that $\sin \left(\theta_{c}-\theta_{12}\right)$ is proportional to $\left|r_{c}-r_{m}\right|^{1 / 2}$, and the singularity in (19) is integrable. This fast change of the angle in the vicinity of $r_{m}$ corresponds to the finite angle change by $-\pi \theta_{0} /\left|\theta_{0}\right|$ when the dipole center crosses the plane center in the case $M=0$ (see above). When $r_{c}=r_{m}$ we see from (21) that $r_{m} r_{12}=|M|$, and the value of $r_{m}$ can be found from (22)

$$
\psi\left(\frac{|M|}{r_{m}}\right)-\psi(1)=\Psi\left(\left|r_{m}+\frac{M}{2 r_{m}}\right|\right)-\Psi\left(\left|r_{m}-\frac{M}{2 r_{m}}\right|\right) .
$$

again since the vortices are aligned radially.

The ratio $r_{m} /|M| \rightarrow 1$ when either $|M| \rightarrow 0$ or $|M| \rightarrow \infty$ as in both cases the right-hand side (hereafter RHS) of (29) vanishes. The RHS of equation (29) can be calculated from equation (24). When $M \Omega_{D}>0$, the RHS of (29) is negative so that $r_{m}<|M|$ and the distance between partners has increased from that at infinity (here $r_{12}>1$ ). When $M \Omega_{D}<0$, the RHS of (29) is positive so that $r_{m}>|M|$ and the distance between partners has decreased $\left(r_{12}<1\right)$. The solutions to (29) for $\gamma=1$ and $r_{D}=0.5$ are shown on Fig.3. For large range of values of $\Omega\left(r_{D}\right)$, there exists a discontinuity of the curve $r_{m}(M)$; this result is confirmed and illustrated by Fig.4 which shows an abrupt change in $r_{m}$ when $M$ decreases and reaches the value indicated on the second plot of Fig.3. This abrupt change in $r_{m}$ indicate the existence of trapped regime when the dipole remain in the vicinity of the seamount to be investigated in a subsequent paper.

After the dipole has reached the point of closest approach of the seamount, $\sin \left(\theta_{c}-\theta_{12}\right)$ becomes positive and the dipole moves away from the plane center, along a trajectory, symmetric with respect to the $\theta_{c}=\theta_{m}$ axis in the phase plane $\left(r_{c}, \theta_{c}\right)$ (indeed $\cos \left(\theta_{c}-\theta_{12}\right)$ does not change sign). Therefore, far from the plane center, the dipole direction approaches $\theta_{c}=2 \theta_{m}$ which is inversely proportional to $U_{\text {eff }}$. 


\section{Conclusion}

This study of the evolution of a point vortex dipole in an axisymmetric flow shows that the motion is integrable, due to the conservation of the Hamitonian and of the angular momentum, which reduces the original four degrees of freedom problem to only two. Thus, the general solution is expressed in quadratures.

For example, we consider the evolution of an antisymmetric dipolar dipole, similar to mushroom-like currents observed in the ocean [9]. For dipoles with zero angular momentum moving towards the center of the plane, the intensity of dipole does not change and its center passes the plane center at some angle proportional to the external rotation rate and inversely proportional to the dipole propagation speed. When the dipole has non-zero angular momentum, it approaches from infinity with an offset from the radial direction. In the vicinity of the plane center, the dipole intensity increases for one sign of the offset and decreases for the other sign of the offset, depending also on the sign of the external rotation. Correspondingly, the dipole center reaches a minimum distance from the plane center, which is either larger or smaller than the offset value. Therefore, farther from the plane center, the dipole may propagate in any direction, with a large sensitivity to the flow parameters (see Fig.5).

For oceanic applications, this investigation can be generalized for a two-layer configuration when dipolar partners in different layers are coupled (the heton model [17]).

\section{acknowledgements}

GS acknowledges support from the NSF Division of Ocean Sciences, the ONR Ocean Science Division, and from Université de Bretagne Occidentale (Brest, France); the hospitality during his visit at Laboratoire de Physique des Oceans was greatly appreciated.

XP was supported by a PhD grant from the French Ministry of Research. 
XC acknowledges support from INTAS (collaborative call with Airbus) under contract 04-80-7297 "Vortex Dynamics" and from Université de Bretagne Occidentale (Brest, France) during the course of this study.

\section{References}

[1] Ruppert-Felsot, J.E., O. Praud, E. Sharon \& H.L. Swinney, 2005. Extraction of coherent structures in a rotating turbulent flow experiment Phys. Rev. E 72, 016311.

[2] Aref H., 1983. Integrable, chaotic, and turbulent vortex motion in twodimensional flows. Annu. Rev. Fluid Mech. 15, 345-89.

[3] Flierl, G.R.,1987. Isolated eddy models in geophysics. Ann. Rev. Fluid Mech. 19, 493-530.

[4] Hopfinger, E.J. \& G. J. F. van Heijst, 1993. Vortices in rotating fluids. Annu. Rev. Fluid Mech. 25, 241-289.

[5] Horton, W. \& A. Hasegawa, 1994. Quasi-two-dimensional dynamics of plasmas and fluids. Chaos 4, 227-251.

[6] Carton, X., 2001. Hydrodynamical modeling of oceanic vortices. Surveys in Geophysics 22, 179-263.

[7] Flierl, G. R., M. E. Stern \& J. A. Whitehead, Jr., 1983. The physical significance of modons: laboratory experiments and general integral constraints. Dyn. Atmos. Oceans. 7, 233-264.

[8] Couder, Y. \& C. Basdevant, 1986. Experimental and numerical study of vortex couples in two-dimensional flows. J. Fluid Mech. 173, 225-251.

[9] Fedorov, K. N. \& A. I. Ginsburg, 1986. "Mushroom-like" currents (vortex dipoles) in the ocean and in a laboratory tank. Adv. Geophys. 4, B, 507-516. [10] Nycander, J. \& M.B. Isichenko, 1990. Motion of dipole vortices in a weakly inhomogeneous medium and related convective transport. Phys. Fluids B 2, $2042-2047$.

[11] Sutyrin, G. G., J. S. Hesthaven, J. P. Lynov, \& J. J. Rasmussen, 1994. Dynamical properties of vortical structures on the beta-plane. J. Fluid Mech. 
268, 103-131.

[12] Trieling, R.R, J.M.A. van Wesenbeeck, \& G.J.F. van Heijst, 1998. Dipolar vortices in a strain flow. Phys. Fluids 10, 144-159.

[13] Bannikova, E.Y., V.M. Kontorovich \& G.M. Reznik, 2007. Dynamics of a vortex pair in radial flow. J. Exp. Theor. Phys. 105, 542-548.

[14] Sutyrin, G.G., 2006. Critical effects of a seamount top on a drifting eddy. J. Mar. Res. 64, 297-317.

[15] Rasmussen, J.J., J.P. Lynov, J.S. Hesthaven \& G.G. Sutyrin, 1994. Vortex dynamics in plasmas and fluids. Plasma Phys. Control. Fusion 36, B193-B202. [16] Sutyrin, G.G \& X. Carton, 2006. Vortex interaction with a zonal Rossby wave in a quasigeostrophic model. Dyn. Atmos. Oceans41, 85-102.

[17] Hogg, N \& H. Stommel, 1985. The heton, an elementary interaction between discrete baroclinic geostrophic vortices and its implication concerning eddy heatflow. Proc. R. Soc. Lond. A 397, 1-20. 

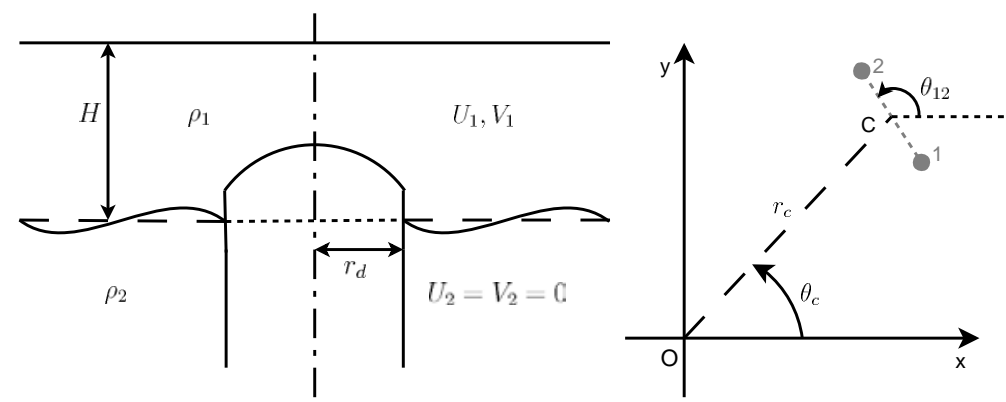

Figure 1: The one and half layer modelisation and the polar system of coordinate.
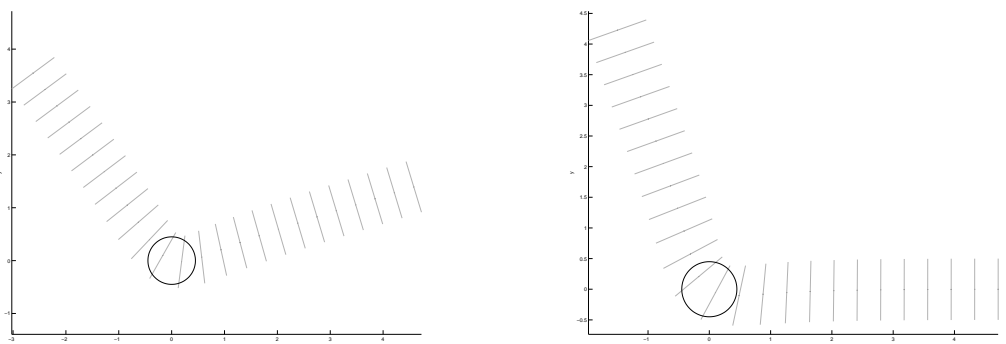

Figure 2: Inflexion of the trajectory of the dipole when it passes the center for different value of $\Omega\left(r_{D}\right)=-0,13$ and $-0,25\left(M=0, r_{D}=0.45\right.$ and $\left.\gamma=1\right)$. The circle represents the position of the seamount, the solid lines link the two point vortices positions for different times and the dots correspond to the center of the solid lines (this convention is used for all others trajectories figures).
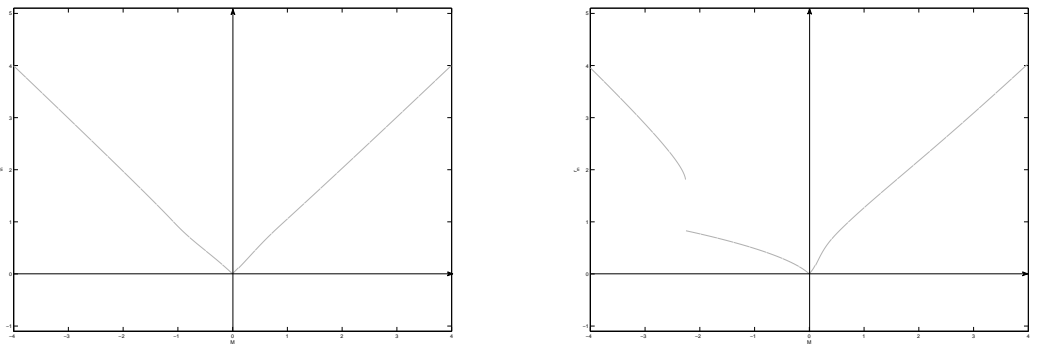

Figure 3: The minimum value of the distance $r_{c}$ between the dipole center and the plane center with respect to $M$ for $\Omega\left(r_{D}\right)=-0,03$ and $-0,25$ 

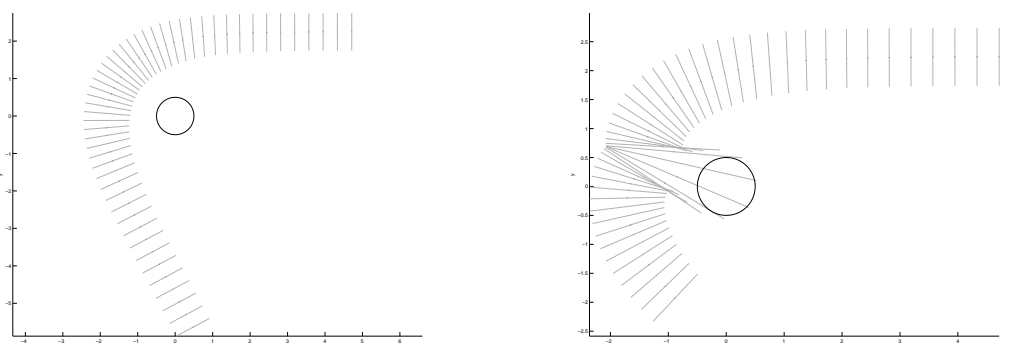

Figure 4: Trajectories of the dipole for $M=-2,25$ and $M=-2,24$ (the time interval is 1)
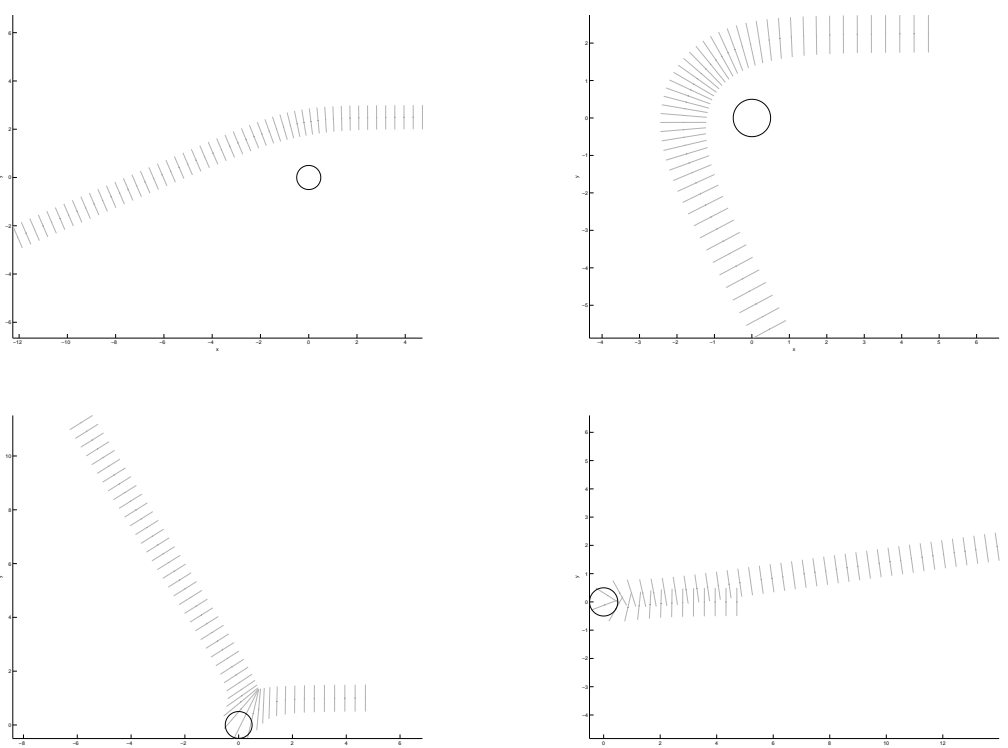

Figure 5: The diversity of dipole trajectories for slightly different initial offsets (from top left to bottom right, decreasing offsets) 